\title{
Genomic Insight into the Salt Tolerance of Enterococcus faecium, Enterococcus faecalis and Tetragenococcus halophilus ${ }^{\text {[s }}$
}

\author{
Sojeong Heo ${ }^{1}$, Jungmin Lee ${ }^{1}$, Jong-Hoon Lee ${ }^{2}$, and Do-Won Jeong ${ }^{1 *}$ \\ ${ }^{1}$ Department of Food and Nutrition, Dongduk Women's University, Seoul, Republic of Korea \\ ${ }^{2}$ Department of Food Science and Biotechnology, Kyonggi University, Suwon, Republic of Korea
}

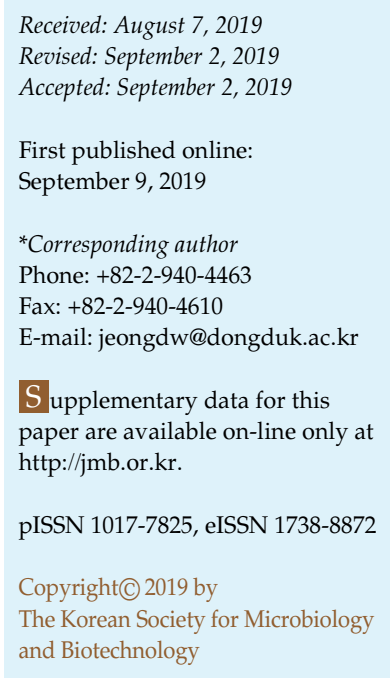

To shed light on the genetic basis of salt tolerance in Enterococcus faecium, Enterococcus faecalis, and Tetragenococcus halophilus, we performed comparative genome analysis of 10 E. faecalis, 11 E. faecium, and three T. halophilus strains. Factors involved in salt tolerance that could be used to distinguish the species were identified. Overall, T. halophilus contained a greater number of potassium transport and osmoprotectant synthesis genes compared with the other two species. In particular, our findings suggested that T. halophilus may be the only one among the three species capable of synthesizing glycine betaine from choline, cardiolipin from glycerol and proline from citrate. These molecules are well-known osmoprotectants; thus, we propose that these genes confer the salt tolerance of T. halophilus.

Keywords: Enterococcus faecium, Enterococcus faecalis, Tetragenococcus halophilus, salt tolerance, pan-genome

\section{Introduction}

Doenjang, a traditional Korean high-salt fermented soybean paste, is made by mixing meju with a high-salt $(\sim 18 \%)$ brine, followed by ripening for approximately 2 months. Meju is a naturally fermented soybean block; microorganisms grow spontaneously during the ripening and produce enzymes that degrade macromolecules in the soybean [1,2]. The microorganisms and enzymes produced enhance the sensory qualities of the product during doenjang production [3-7]. Doenjang is used frequently in Korean cuisine and has thus been the subject of several studies, including of the microbial community. In initial microbial studies, the presence of fungal species belonging to the genera Mucor, Penicillium, Scopulariopsis, Aspergillus, Rhodotorula, Torulopsis (amended to Candida), and Saccharomyces was confirmed using culture-dependent methods, as was the presence of bacterial species belonging to the genus Bacillus and lactic acid bacteria such as Lactobacillus sp. [810]. Recent culture-independent analysis methods such as pyrosequencing [11] have shown that lactic acid bacteria, including Enterococcus and Tetragenococcus, are predominant bacteria in doenjang, alongside Bacillus [12-16].

The genera Enterococcus and Tetragenococcus are included in the family Enterococcaceae [17]. Enterococcus species have been detected in fermented foods such as dairy foods [18-20], while Tetragenococcus species are widely detected in high-salt-fermented food products including fish, soy pastes, and soy sauce, and are considered potential starters for the production of these foods [21]. Although 16S rRNA gene sequences of species from the two genera are very similar [22], Enterococcus faecalis and Enterococcus faecium cannot grow in medium with $\mathrm{NaCl}$ concentrations greater than $7 \%(\mathrm{w} / \mathrm{v})$, while Tetragenococcus halophilus can grow in media with $\mathrm{NaCl}$ concentrations of up to $21 \%$ (w/v) [23-25].

Recently, we examined the diversity of cultivable bacteria using media supplemented with $\mathrm{NaCl}$ to understand changes in the bacterial community during processing from meju to doenjang [23]. E. faecalis and E. faecium, together with bacilli and coagulase-negative staphylococci, were the predominant bacterial species during meju fermentation. However, the community composition shifted to include 
salt-tolerant bacilli, coagulase-negative staphylococci, and T. halophilus as the predominant members during doenjang fermentation, which involved inoculation via sea salt during the brining process. These results showed that Enterococcus sp. and T.halophilus are candidate starter species for meju and doenjang, respectively.

We have also assessed the safety and technological properties of E. faecium, E. faecalis, and T. halophilus strains from fermented soybeans to select functional and safe starter candidates [24-26]. The selected starter candidates (E. faecium and T. halophilus) were inoculated into sterilized soybeans to assess their contributions to the sensory properties of the products [6]. Our results showed that E. faecium and T. halophilus produced a similar profile of volatile compounds in soybeans, with no dramatic differences in soybean flavor. However, no comprehensive picture of the cellular components and metabolic pathways involved in the degradation of macromolecules and the development of sensory properties by E. faecium and T. halophilus during soybean fermentation has been obtained. In addition, currently available genomic data are insufficient to identify the genomic features of various strains that contribute to differences in salt tolerance $[22,27]$.

In the current study, we performed a comparative genomic analysis of Enterococcus and Tetragenococcus species to define the scale and scope of the pan-genome and to identify the core genes, as well as clarify the genetic background of strains from different niches, with particular reference to salt tolerance.

\section{Materials and Methods}

\section{Comparative Genomics}

For comparative genomic analysis, genome sequence data for 10 E. faecalis, 11 E. faecium, and three T. halophilus strains were obtained from the NCBI genome database (http://ncbi.nlm.nih. gov/genomes) (Table 1). T. halophilus KUD23 was isolated and selected as a starter candidate for the fermentation of high-salt foods in our previous study [26]. Average nucleotide identity, which provides a robust measurement of genetic distance among bacterial genomes, was used for comparative analysis of the conserved genes among the genomes [28]. Genome sequences of the 24 strains were uploaded to the Rapid Annotations using the Subsystems Technology server for SEED-based automated annotation, whole-genome sequence-based comparative analysis, and Kyoto Encyclopedia of Genes and Genomes metabolic pathway analysis [29]. The efficient database framework for comparative genome analyses using basic local alignment search tool (BLAST) score ratios was used for core genome, pan-genome, and singleton analyses [30]. The genome of T. halophilus KUD23 was used as a reference genome for Venn diagram construction. Further comparative analyses were performed for specific regions and genes of interest using the BLASTN, BLASTX, and BLASTP tools.

\section{Growth in the Presence of $\mathrm{NaCl}$}

E. faecalis KCTC 2011, E. faecium KCCM 12118, and T. halophilus KUD23 were analyzed for growth in the presence of $3.5 \%, 7 \%$, or $14 \%(\mathrm{w} / \mathrm{v}) \mathrm{NaCl}$. In addition, the three strains were assayed for growth on tryptic soy agar (TSA; Difco, USA) containing 7\% (w/v) $\mathrm{NaCl}$ and $0.25 \%(\mathrm{w} / \mathrm{v})$ choline, citrate, glycerol, or glycine betaine. Strains were cultured in tryptic soy broth (TSB; Difco), normalized to a turbidity of 1.0 at an optical density of $600 \mathrm{~nm}$, and then diluted 1:10 in fresh TSB. A 10- $\mu$ l aliquot of diluted sample was then dropped onto the surface of a TSA plate supplemented with $\mathrm{NaCl}$ at concentrations of $3.5 \%, 7 \%$, or $14 \%(\mathrm{w} / \mathrm{v})$. Cell growth was then assessed following incubation at $30^{\circ} \mathrm{C}$ for $48 \mathrm{~h}$.

\section{Results and Discussion}

\section{Genome Summary and General Features}

The general features of the genomes of the 24 strains are summarized in Table 1 . The average genome sequence lengths of the $10 \mathrm{E}$. faecalis, $11 \mathrm{E}$. faecium, and three T. halophilus strains were 3,018,574, 3,045,285, and 2,517,102 bp, respectively. Among the strains, T. halophilus strain MJ4 from the high-salt fermented food myeolchi-jeot (pickled anchovy), had the smallest genome $(2,389,470 \mathrm{bp})$, while E. faecium strain $6 \mathrm{E} 6$ from human feces had the largest genome $(3,397,850 \mathrm{bp})$. The average $\mathrm{G}+\mathrm{C}$ content percentages of the E. faecalis, E. faecium, and T. halophilus genomes were $37.44 \%, 37.97 \%$, and 36.3\%, respectively. All T. halophilus genomes displayed a similar $\mathrm{G}+\mathrm{C}$ content, which was low compared with the other two species.

To allow a coherent comparative analysis, we performed consistent open reading frame (ORF) predictions for the complete genome sequences of the 24 strains. An average of 2,987, 3,042, and 2,393 ORFs were identified in the E. faecalis, E. faecium, and T. halophilus genomes, respectively (Table 1). Notably, BLAST-based functional in silico prediction was achieved for $86.2 \%, 85.5 \%$, and $96.3 \%$ of the identified ORFs in E. faecalis, E. faecium, and T. halophilus, respectively.

Analysis using Clusters of Orthologous Groups (COG) functional categorization and SEED subsystem categorization predicted the existence of an average of 2,576 coding sequences (CDSs) and 2,056 CDSs, respectively, in E. faecalis, 2,599 and 1,885 CDSs, respectively, in E. faecium, and 2,305 and 1,747 CDSs, respectively, in T. halophilus. Based on COG functional categorization, genes involved in carbohydrate transport and metabolism (average 10.2\%) were the most 
Table 1. General genomic and specific phenotypic features of 24 lactic acid bacterial strains.

\begin{tabular}{|c|c|c|c|c|c|c|c|c|c|c|c|c|c|c|c|c|}
\hline Species & Strain & $\begin{array}{l}\text { Ref } \\
\text { No. }\end{array}$ & Size (bp) & $\begin{array}{l}\text { Chromosome } \\
\text { size (bp) }\end{array}$ & $\begin{array}{c}\mathrm{G}+\mathrm{C} \\
\text { content } \%\end{array}$ & $\begin{array}{l}\text { No. of } \\
\text { plasmids }\end{array}$ & COG & SEED & $\begin{array}{l}\text { No. of } \\
\text { rRNAs }\end{array}$ & $\begin{array}{l}\text { No. of } \\
\text { tRNAs }\end{array}$ & $\begin{array}{l}\text { Other } \\
\text { RNAs }\end{array}$ & Contigs & Origin & Country & Accession No. & Status \\
\hline \multirow{10}{*}{$\begin{array}{l}\text { Enterococcus } \\
\text { faecalis }\end{array}$} & 62 & $\mathrm{~A} 1$ & $3,130,820$ & $2,988,673$ & 37.36 & 3 & 2,667 & 2,071 & 12 & 54 & 4 & 5 & Healthy Norwegian infant & Norway & СР002491 & Complete \\
\hline & ATCC 29212 & A2 & $3,048,130$ & $2,939,972$ & 37.36 & 2 & 2,571 & 2,078 & 12 & 61 & 4 & 3 & Homo sapiens urine & USA & NZ_CP008816 & Complete \\
\hline & CLB21560 & A3 & $3,243,540$ & $3,111,017$ & 37.08 & 2 & 2,737 & 2,112 & 12 & 66 & 4 & 3 & Homo sapiens clinical & USA & NZ_CP019512 & Complete \\
\hline & D32 & A4 & $3,062,500$ & $2,987,450$ & 37.44 & 2 & 2,594 & 2,037 & 12 & 54 & 4 & 3 & Pig feces & Germany & NC_018221 & Complete \\
\hline & DENG1 & A5 & $2,961,043$ & $2,961,043$ & 37.50 & - & 2,581 & 2,061 & 12 & 62 & 4 & 1 & Sputum of 54-year-old male & China & NZ_CP004081 & Complete \\
\hline & KB1 & A6 & $3,026,009$ & $3,026,009$ & 37.20 & - & 2,526 & 2,024 & 12 & 59 & 4 & 1 & Mus musculus & Germany & NZ_CP015410 & Complete \\
\hline & LD33 & A7 & $2,803,429$ & $2,803,429$ & 37.60 & - & 2,459 & 2,047 & 12 & 61 & 4 & 1 & Traditional dairy product & China & NZ_CP014949 & Complete \\
\hline & OG1RF & A8 & $2,739,625$ & $2,739,625$ & 37.80 & - & 2,330 & 1,950 & 12 & 58 & 4 & 1 & Patient with endocarditis & USA & NC_017316 & Complete \\
\hline & Symbioflor 1 & A9 & $2,810,675$ & $2,810,675$ & 37.70 & - & 2,469 & 2,013 & 12 & 63 & 4 & 1 & Healthy human adult & Germany & NC_019770 & Complete \\
\hline & V583 & A10 & $3,359,970$ & $3,218,031$ & 37.35 & 3 & 2,822 & 2,167 & 12 & 59 & 4 & 4 & Blood of a hospitalized patient & USA & NC_004668 & Complete \\
\hline \multirow{11}{*}{$\begin{array}{l}\text { Enterococcus } \\
\text { faecium }\end{array}$} & 2014-VREF-41 & I1 & $3,280,730$ & $3,009,007$ & 37.59 & 4 & 2,749 & 1,893 & 18 & 68 & 4 & 5 & Homo sapiens rectal swab & Korea & NZ_CP019208 & Complete \\
\hline & $64 / 3$ & I2 & $2,572,333$ & $2,572,333$ & 38.20 & - & 2,205 & 1,859 & 18 & 68 & 4 & 1 & Homo sapiens & Germany & NZ_CP012522 & Complete \\
\hline & $6 \mathrm{E} 6$ & $\mathrm{I} 3$ & $3,397,850$ & $2,966,909$ & 37.60 & 2 & 2,874 & 1,893 & 18 & 75 & 4 & 3 & Homo sapiens feces & USA & NZ_CP013994 & Complete \\
\hline & ATCC 700221 & I4 & $3,151,410$ & $2,859,123$ & 38.10 & 3 & 2,843 & 2,031 & 18 & 72 & 4 & 4 & Homo sapiens feces & USA & СР014449 & Complete \\
\hline & Aus0004 & I5 & $3,019,780$ & $2,955,294$ & 38.34 & 3 & 2,565 & 1,895 & 18 & 47 & 4 & 4 & Homo sapiens blood & Australia & NC_017022 & Complete \\
\hline & E39 & I6 & $3,108,030$ & $2,790,106$ & 37.82 & 5 & 2,611 & 1,772 & 18 & 70 & 4 & 6 & Blood of a hospitalized patient & USA & NZ_CP011281 & Complete \\
\hline & ISMMS_VRE_1 & I7 & $3,259,290$ & $3,130,373$ & 37.67 & 5 & 2,807 & 1,997 & 18 & 72 & 4 & 6 & Homo sapiens blood & USA & NZ_CP012430 & Complete \\
\hline & NRRL B-2354 & I8 & $2,849,890$ & $2,635,572$ & 37.85 & 1 & 2,438 & 1,882 & 18 & 48 & 4 & 2 & Dairy utensils & USA & NC_020207 & Complete \\
\hline & $\mathrm{T} 110$ & I9 & $2,737,960$ & $2,693,877$ & 38.46 & 1 & 2,299 & 1,838 & 18 & 65 & 4 & 2 & Medical & India & NZ_CP006030 & Complete \\
\hline & UW8175 & I10 & $2,878,190$ & $2,598,959$ & 38.02 & 3 & 2,472 & 1,808 & 18 & 66 & 4 & 4 & Homo sapiens & Germany & NZ_CP011828 & Complete \\
\hline & VRE001 & I11 & $3,242,670$ & $2,933,966$ & 37.81 & 3 & 2,731 & 1,871 & 18 & 72 & 4 & 4 & Homo sapiens blood & USA & NZ_CP018071 & Complete \\
\hline \multirow{3}{*}{$\begin{array}{l}\text { Tetragenococcus } \\
\text { halophilus }\end{array}$} & KUD23 & $\mathrm{T} 1$ & $2,599,117$ & $2,599,117$ & 36.10 & - & 2,390 & 1,795 & 15 & 62 & 4 & 1 & Doenjang & Korea & NZ_CP020017 & Complete \\
\hline & MJ4 & $\mathrm{T} 2$ & $2,389,470$ & $2,389,470$ & 36.00 & - & 2,173 & 1,689 & 15 & 62 & 4 & 1 & Myeolchi-jeot & Korea & NZ_CP012047 & Complete \\
\hline & NBRC 12172 & $\mathrm{~T} 3$ & $2,562,720$ & $2,562,720$ & 36.00 & - & 2,351 & 1,756 & 15 & 62 & 4 & 1 & Soy sauce brewing & Japan & NC_016052 & Complete \\
\hline
\end{tabular}




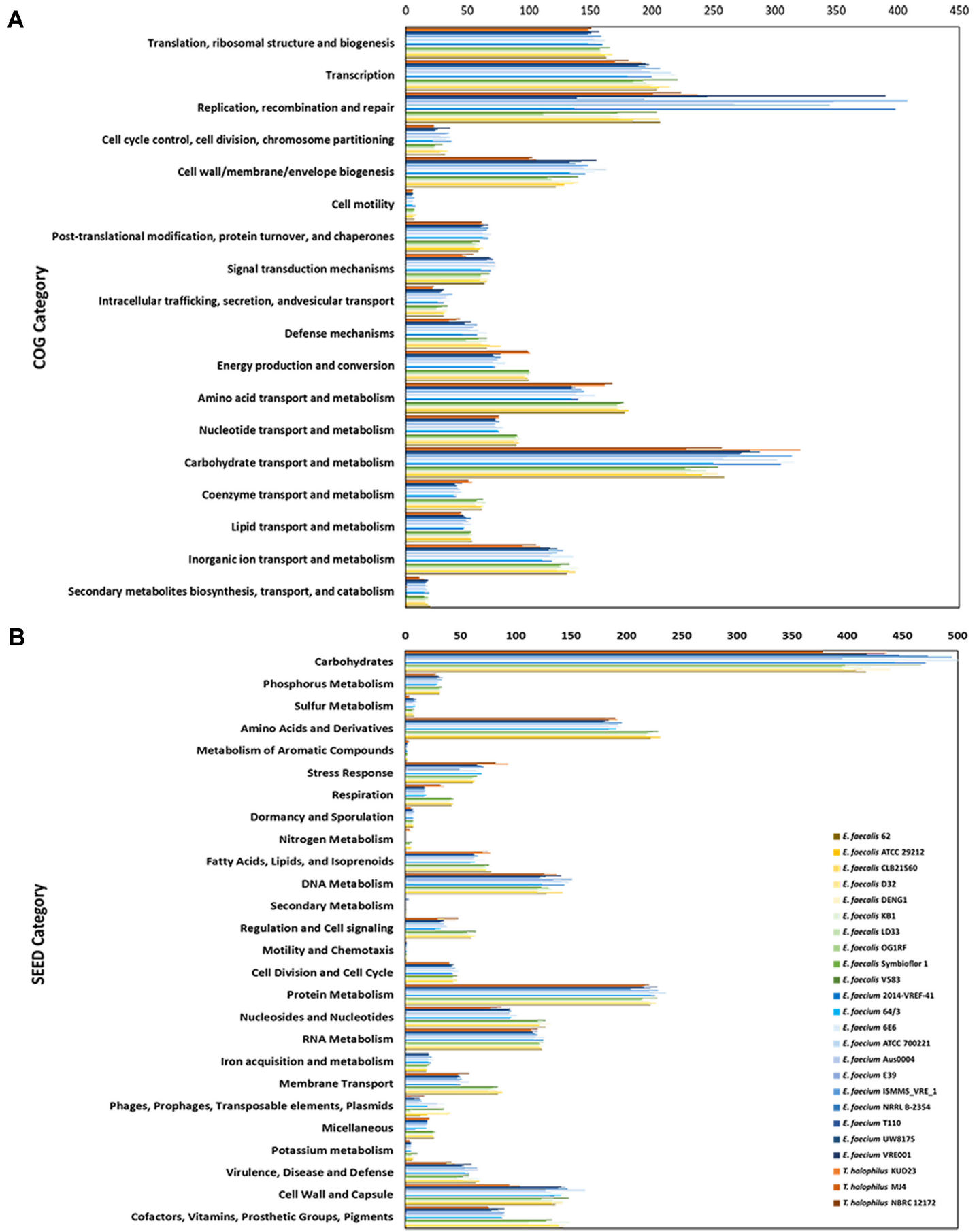

Fig. 1. Average numbers of genes in functional categories in 10 E. faecalis, 11 E. faecium, and three T. halophilus genomes based on Clusters of Orthologous Groups (COG) (A) and SEED (B) analyses.

Genome sequences of 24 strains were independently uploaded to the COG and SEED viewer servers. Functional roles of annotated genes were assigned and grouped in subsystem feature categories. Colored bars indicate the number of genes assigned to each category.

abundant category in E. faecalis, followed by those required for transcription (average 8.5\%). Genes required for replication, recombination, and repair (average 12.3\%) and carbohydrate transport and metabolism (average 12.5\%) were the most abundant categories in E. faecium and T. halophilus, respectively. Based on the SEED subsystem, a 
large number of genes from all three species were allocated to protein metabolism $(10.7 \%-12.5 \%)$ and amino acid biosynthesis and utilization $(9.9 \%-10.9 \%)$ (Fig. 1). Although the two types of analysis showed different percentages among the three species, the major functional groups were similar.

\section{Comparative Analysis of the E. faecalis, E. faecium, and} T. halophilus Genomes

For whole-genome comparison of the 10 E. faecalis, 11 E. faecium, and three T. halophilus strains, we analyzed the core and pan-genomes of the different species. Genes shared across all E. faecalis strains decreased with each addition, finally reaching a plateau around 2,007 genes (Fig. 2), while the pan-genome predicted at least 4,818 genes. Analysis of the 11 E. faecium strains resulted in core and pan-genomes of approximately 1,652 and 4,461 genes, respectively. The core genome accounted for approximately $63.6 \%-77.9 \%$ of all genes in each genome. Because of the limited number of available genomes, it was not possible to determine core and pan-genomes for T. halophilus.

Common strain-specific genes among the pan-genomes of each species included phage transferable elements such as relaxase, genes encoded on plasmids, and genes encoding hypothetical proteins (Table S1). Our findings suggested variability in gene content between species, as well as among strains of the same species. These results once again implied genomic plasticity among species living in different habitats with diverse lifestyles. An open pan-genome is typical of species that colonize multiple environments and have various ways of exchanging genetic material.

We next analyzed the genes shared among three representative species from food-E. faecalis LD33 from a traditional dairy product from China, E. faecium NRRL B2354 from dairy utensils, and T. halophilus KUD23 from fermented soybean (Fig. 3 and Table S1). The three strains shared 1,084 CDSs within the core genome, corresponding to approximately $44.08 \%-45.36 \%$ of all ORFs. We assume that this core genome is very small because of low genomic similarity among these three representative strains. Most CDSs in the core genome were assigned functions via COG annotation that related to metabolism and the transport of amino acids and carbohydrate utilization. The majority of strain-specific genes were associated with hypothetical proteins, phage proteins, or specific plasmids. Other functional strain-specific genes in the genomes of strains LD33, NRRL B-2354, and KUD23 included streptococcinencoding genes, tetracycline resistance major facilitator superfamily efflux protein-encoding genes, and CRISPRassociated protein-encoding genes, respectively. Unique genes in T. halophilus were related to potassium transport, glycine/betaine transport, an aspartate-alanine antiporter, an anion permease, and electron transport. These T. halophilusspecific genes were detected in all three examined T. halophilus genomes. These results implied that the higher salt-tolerance of T. halophilus compared with the Enterococcus species might be associated with these transporters.

\section{E. faecalis 10 strains}

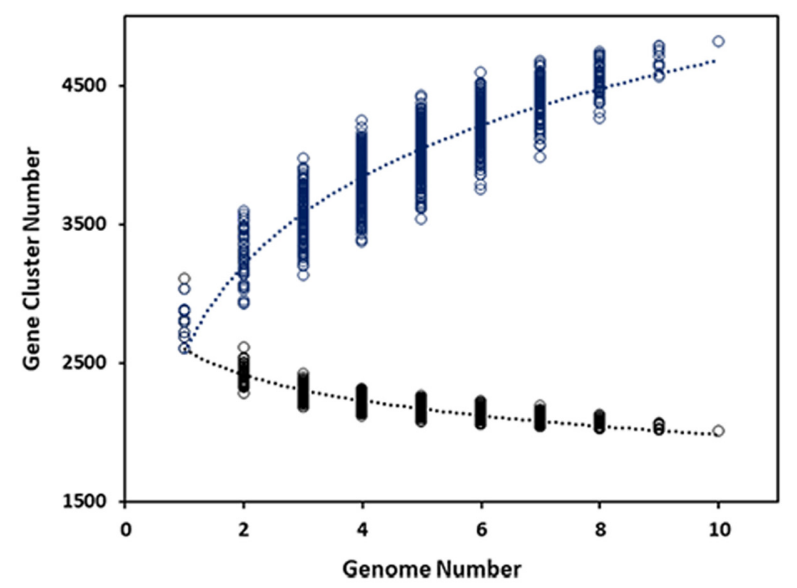

\section{E. faecium 11 strains}

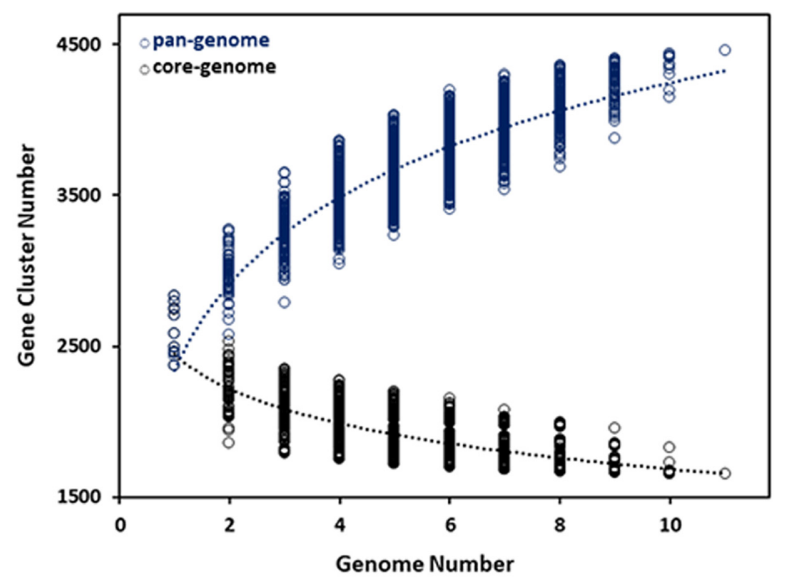

Fig. 2. Sizes of the core and pan-genomes of the 10 E. faecalis and 11 E. faecium strains.

The black (core genome) and blue (pan-genome) curves were fitted to the decay function $(916.810 \times \exp (-\mathrm{x} / 3.226)+2010.866$ for E. faecalis and $904.475 \times \exp (-\mathrm{x} / 4.889)+1589.834$ for E. faecium $)$ and Heap's law function $\left(2503.183 \times \mathrm{x}^{0.236}\right.$ for E. faecalis and $2402.594 \times \mathrm{x}^{0.463}$ for E. faecium), respectively. Each dot shows the gene cluster number of the individual genome. 


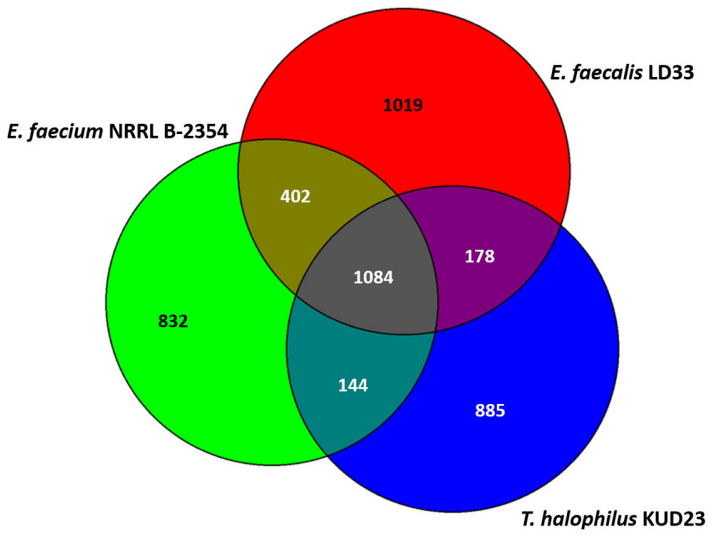

Fig. 3. Venn diagram of E. faecalis, E. faecium, and T. halophilus genomes.

Venn diagram generated using the Efficient Database framework for comparative Genome Analyses using BLAST score Ratios (EDGAR). Overlapping regions represent coding sequences (CDSs) shared between species genomes. The numbers outside the overlapping regions indicate the numbers of CDSs in each genome without homologs in the other species.

\section{Insights into Salt Tolerance}

Bacteria respond to osmotic pressure by accumulating or releasing solutes [31]. Relevant solutes include inorganic ions such as $\mathrm{K}^{+}$, along with organic molecules known as osmolytes. Therefore, we focused our analysis on genes related to the synthesis and transport of these solutes to explain the differences in salt tolerance between the three species.

Inorganic molecules. T. halophilus is better able to survive high-salt conditions than many other foodassociated bacteria, including E. faecalis and E. faecium [2325]. However, the mechanism that allows T. halophilus to tolerate such high-salt conditions is not well understood. Potassium $\left(\mathrm{K}^{+}\right)$plays a pivotal role in, and is the most abundant ion in the bacterial cytoplasm [32]. It is required for the activity of intracellular enzymes and is involved in the maintenance of a constant internal $\mathrm{pH}$ and membrane potential. Potassium also has an important function as an osmotic solute. Many bacteria, including halophiles, accumulate potassium ions within the cell in response to increases in external $\mathrm{NaCl}$ concentrations, although compatible organic solutes are preferred [33,34]. A rapid import of potassium ions is triggered in Gram-positive bacterium Bacillus subtilis in response to osmotic upshock [35]. Holtmann et al. also reported that potassium ion concentrations decreased under osmotic downshock [36]. Bacteria usually express multiple specific uptake systems involved in the adjustment of potassium ion concentration.
We hypothesized that the T. halophilus genome would contain more genes related to potassium transport than are found in the genomes of the two less salt-tolerant Enterococcus species. As shown in Fig. 4, we identified potassium transporter genes in the genomes of all three species. T. halophilus contained two potassium transport systems-a Trk potassium uptake family system and potassium transporter $\mathrm{YbaL}$-while E. faecalis and E. faecium only contained the genes for the Trk system. Based on the pan-genome analysis, T. halophilus was predicted to contain a greater number of potassium uptake genes than the other strains (Table S1); however, this was not supported by EC number matching of specific genes and direct gene similarity analysis.

Organic molecules. To achieve salt tolerance, bacterial proteins undergo extensive amino acid substitution with aspartyl, glutamyl, and weakly hydrophobic residues [37]. Solute accumulation stimulates bacterial growth at high osmotic pressure, and solute release allows cells to survive osmotic downshock. Studies of bacterial osmoregulation have focused on enzymes, transporters, and channels mediating solute accumulation and release.

Compatible solutes such as glycine betaine, proline betaine and carnitine accumulation can be accomplished through biosynthesis and/or transport from the environment. Representative transporters of compatible solute in B. subtilis are osmoprotectant uptake (Opu) systems [38]. All three species examined in the current study commonly contained three Opu systems, OpuB, OpuC, and OpuD, while only $T$. halophilus contains the additional OpuA system (Fig. 4). While OpuA, OpuB, and OpuC belong to the ATP-binding cassette transporter superfamily, OpuD is a single-component transporter belonging to the BCCT family; however, all Opu import systems are known to be osmotically regulated [38]. Chun et al. recently showed that T. halophilus contains the OpuA and OpuC systems [39], while Lin et al. reported that OpuA (BusA) plays an important role in adaptation to high-salt conditions [40]. In the current study, only the T. halophilus strains contained all of the genes required for a functional OpuA system, with the E. faecalis and E. faecium strains missing the solutebinding, protein-encoding gene (Fig. 4). Overall, our analysis showed that the T. halophilus strains possessed four Opu systems (OpuA, OpuB, OpuC, and OpuD), while the E. faecalis and E. faecium species only contained OpuB, OpuC, and OpuD (Fig. 4). Therefore, we suggest that T. halophilus has an advantage in the uptake of compatible solutes, not only glycine betaine but also proline betaine, compared with E. faecalis and E. faecium. 


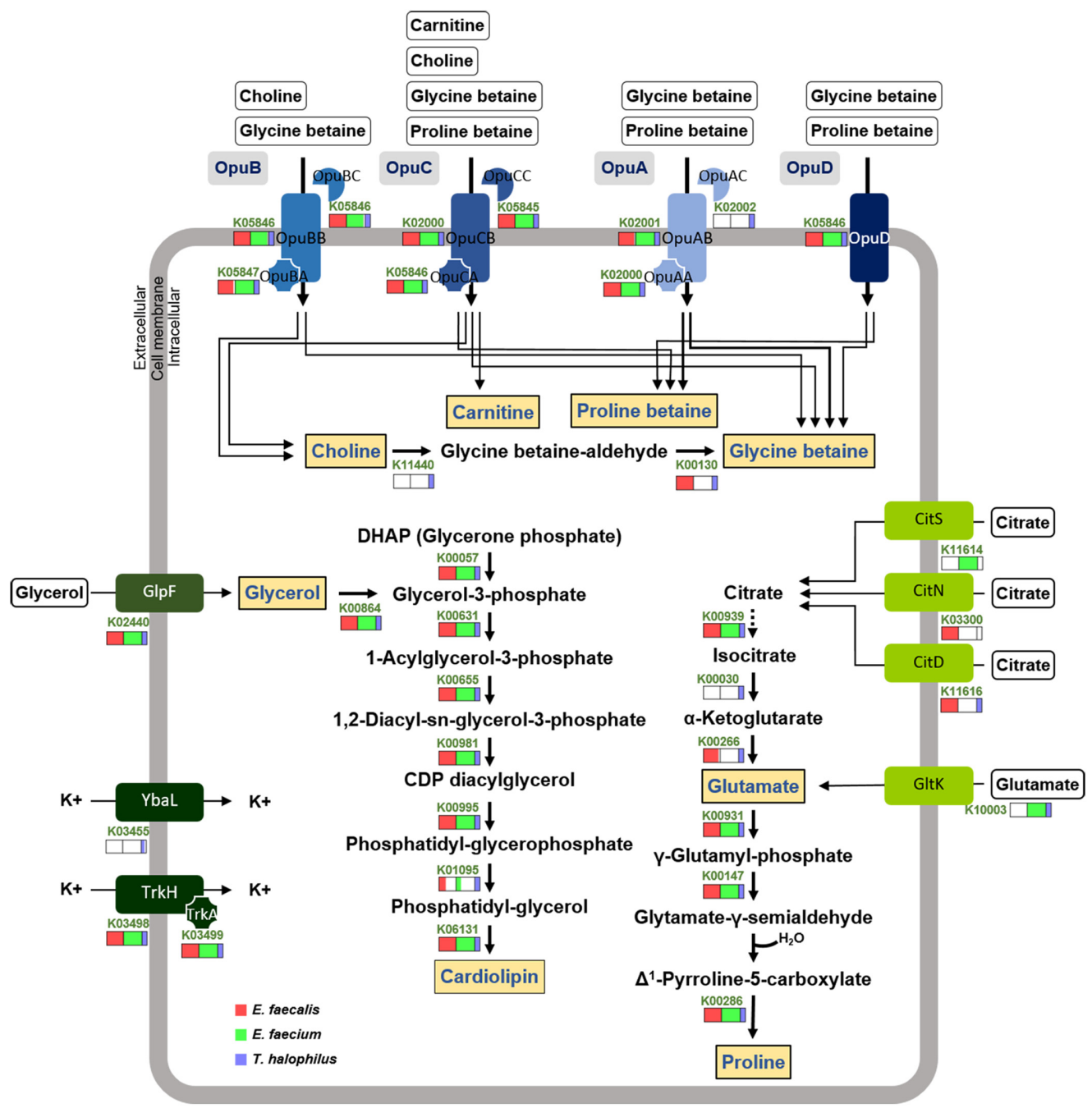

Fig. 4. Predicted membrane transport systems and synthesis pathways for osmoprotectants in the E. faecalis, E. faecium, and T. halophilus genomes.

Enzymes are marked with suggested EC numbers or KEGG numbers. Osmoprotectants are depicted in orange. Black arrows correspond to potential enzymatic reactions catalyzed by gene products encoded by the three genomes.

Glycine betaine is one of the most potent and widely used compatible solutes in nature. Metabolic analysis of T. halophilus under high-salt conditions suggested that glycine betaine was the main compatible solute in this species [39]. The precursor of glycine betaine, choline, must be taken up from exogenous sources via the Opu transporters. The abilities of these systems to transport choline have been confirmed previously in B. subtilis [38]. The presence of four high-affinity, osmotically-regulated choline and/or glycine betaine transporters therefore gives T. halophilus an advantage over the other two species with reference to choline uptake. All of the T. halophilus strains also contained genes encoding a soluble type III alcohol dehydrogenase ( $g b s B ; \mathrm{K} 11440)$ and a glycine betaine aldehyde dehydrogenase $(g b s A ; \mathrm{K} 00130)$, both of which are required for the synthesis of glycine betaine from choline [41] (Fig. 4). However, $g b s B$ was not identified in any of the E. faecalis or E. faecium genomes, although choline transporter genes were detected (Fig. 4). Although it appears that glycine betaine cannot be synthesized from choline in E. faecalis and E. faecium, three glycine betaine transporters were detected in these species, and glycine betaine enhanced the growth of E. faecalis and E. faecium on TSA plates containing $7 \% \mathrm{NaCl}$. Choline also increased the growth of the two species, although to a lesser extent than glycine betaine (Fig. 5). 
A

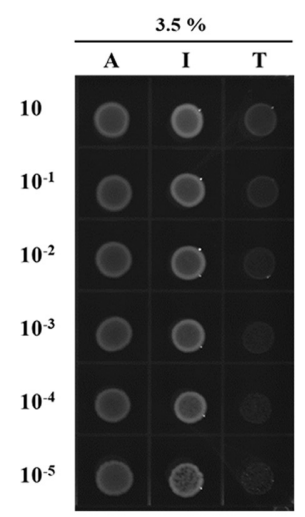

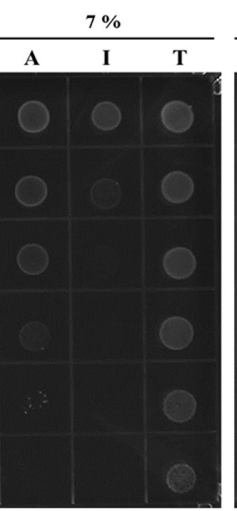

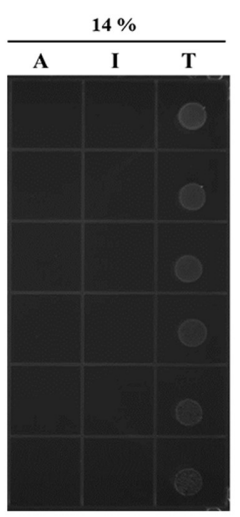

B

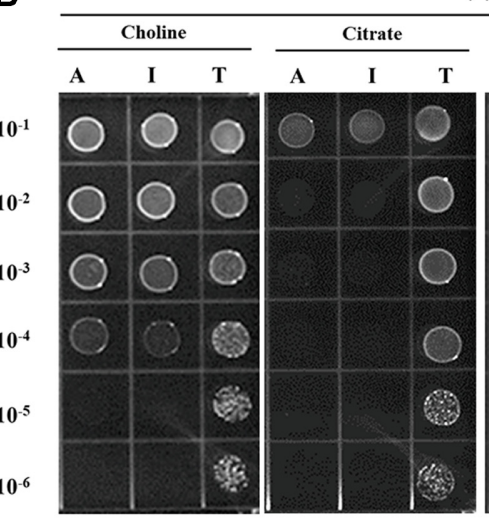

$7 \%$

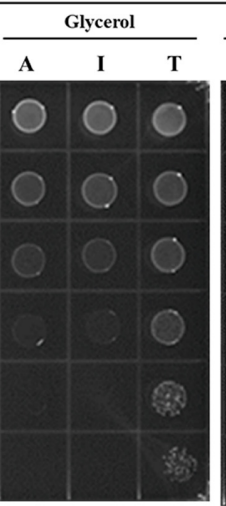

Glycine betaine

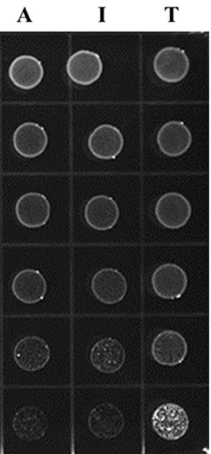

Fig. 5. Growth of E. faecalis, E. faecium, and T. halophilus in tryptic soy agar supplemented with $\mathrm{NaCl}$ at different concentrations (A) or with various osmoprotectants and $\mathrm{NaCl}(7 \%, \mathrm{w} / \mathrm{v})(\mathbf{B})$.

Abbreviations: A, E. faecalis KCTC 2011; I, E. faecium KCCM 12118; T, T. halophilus KUD23.

The lipid cardiolipin also plays a role in adaptation to high salinity stress $[42,43]$, and was shown to be necessary for the prolonged survival of Staphylococcus aureus under high-salt conditions $[43,44]$. Staphylococcus equorum strains containing cardiolipin synthesis genes also showed salt tolerance [45]. Cardiolipin is synthesized from glycerol-3phosphate (Fig. 4), and our analysis revealed that T. halophilus, E. faecalis, and E. faecium all contained genes allowing the synthesis of glycerol-3-phosphate from glucose and glycerol. The complete cardiolipin synthesis pathway was present in all of the examined T. halophilus strains, four E. faecalis strains, and three E. faecium strains. These results imply that cardiolipin synthesis is strain-specific in E. faecalis and E. faecium species. To determine whether the addition of glycerol improved the salt tolerance of the three examined bacterial species, potentially indicating the synthesis of cardiolipin, the growth of E. faecalis KCTC 2011, E. faecium KCCM 12118, and T. halophilus KUD23 was assessed on TSA supplemented with $7 \% \mathrm{NaCl}$ and $0.25 \%$ glycerol (Fig. 5B). Glycerol increased the growth of all three strains, suggesting that glycerol was used as an osmoprotectant. However, it remains unclear whether cardiolipin was synthesized from glycerol.

Proline is another compatible solute. T. halophilus and the two Enterococcus species did not possess a proline transporter, but did contain genes for the biosynthesis of proline from glutamate or citrate (Fig. 4). Glutamate is also a compatible solute, and a specific glutamate transporter was found in E. faecium and T. halophilus. However, only the T. halophilus strains contained a dedicated biosynthesis pathway for the production of glutamate from citrate, although most of the examined strains from all three species possessed a citrate transporter (Table 2). Metabolic analysis of T. halophilus under high-salt conditions revealed a decrease in the activity of the glutamate importer [39], assuming that proline may be synthesized from citrate. Accumulation of intracellular proline in T. halophilus led to protect cells against salt stress [46]. Therefore, we hypothesize that $T$. halophilus has an advantage over the other two species in the synthesis of proline as a compatible solute. In addition, citrate did not enhance the growth of E. faecalis or E. faecium compared with that of T. halophilus on TSA containing $7 \% \mathrm{NaCl}$, although these results are not direct evidence of proline synthesis from citrate (Fig. 5A).

We hypothesized that the $T$. halophilus genome would contain transporter systems and/or compatible solute biosynthesis genes that distinguished it from the Enterococcus species. Comparative genomic analysis confirmed that the T. halophilus genome contains a greater number of transporter systems and compatible solute biosynthesis genes than are found in the genomes of the Enterococcus species. These results suggest that $T$. halophilus has an advantage in the uptake of compatible solutes such as glycine betaine and in the synthesis of solutes such as cardiolipin and proline compared with E. faecalis and E. faecium, although it did not possess distinctive salt tolerance genes.

Based on comparative genomic analysis, we predicted that E. faecalis would be more salt tolerant than E. faecium. To test this, we examined the growth of E. faecalis KCTC 2011, E. faecium KCCM 12118, and T. halophilus KUD23 on TSA supplemented with $3.5 \%, 7 \%$, or $14 \%(\mathrm{w} / \mathrm{v}) \mathrm{NaCl}$ (Fig. 5B). Overall, the growth rates matched our predictions based on genomic analysis: E. faecalis contained more genes required for the uptake of compatible solutes compared 
Table 2. Osmoprotectant synthesis- and transporter system-related genes in the genomes analyzed in this study.

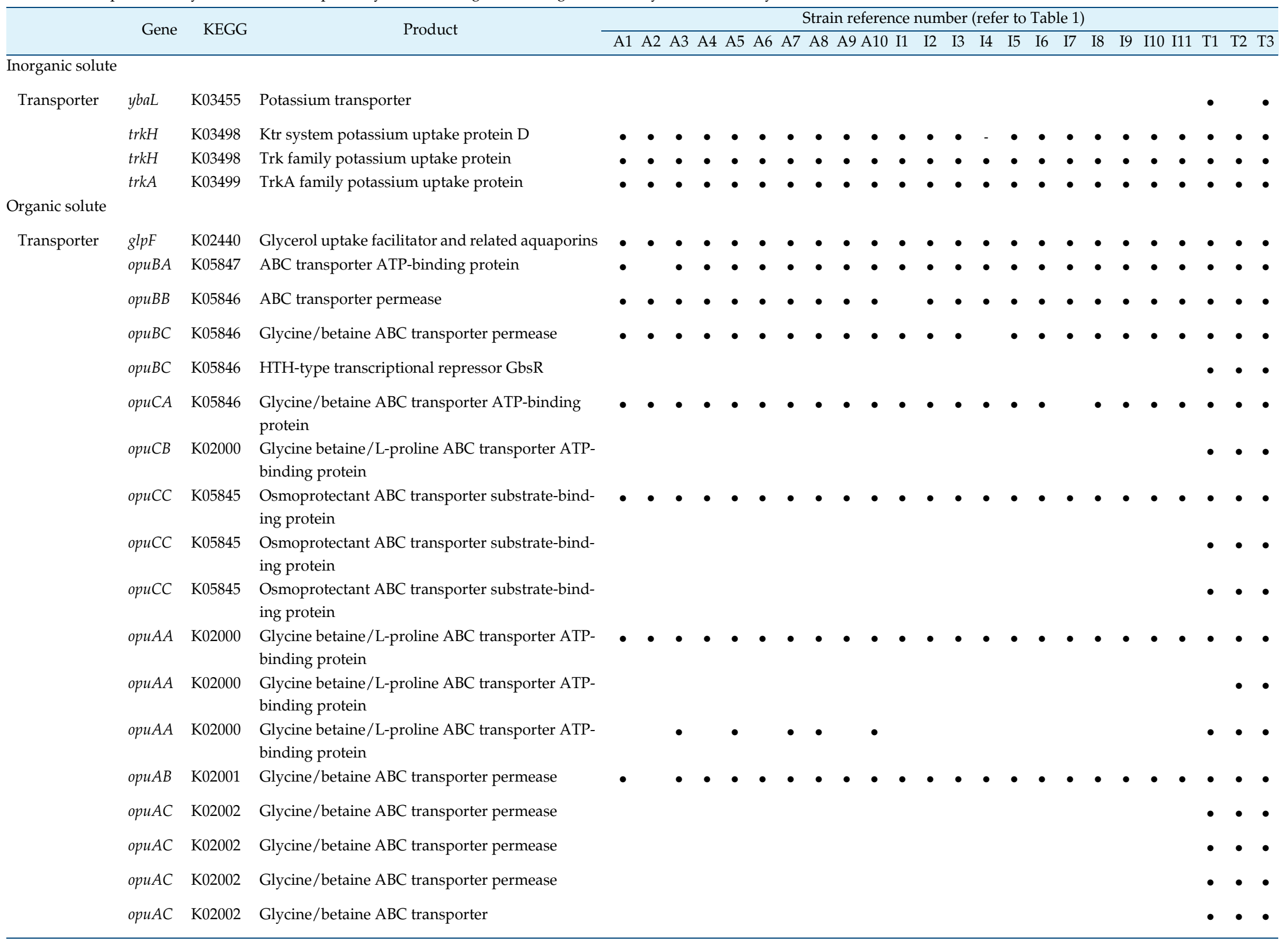




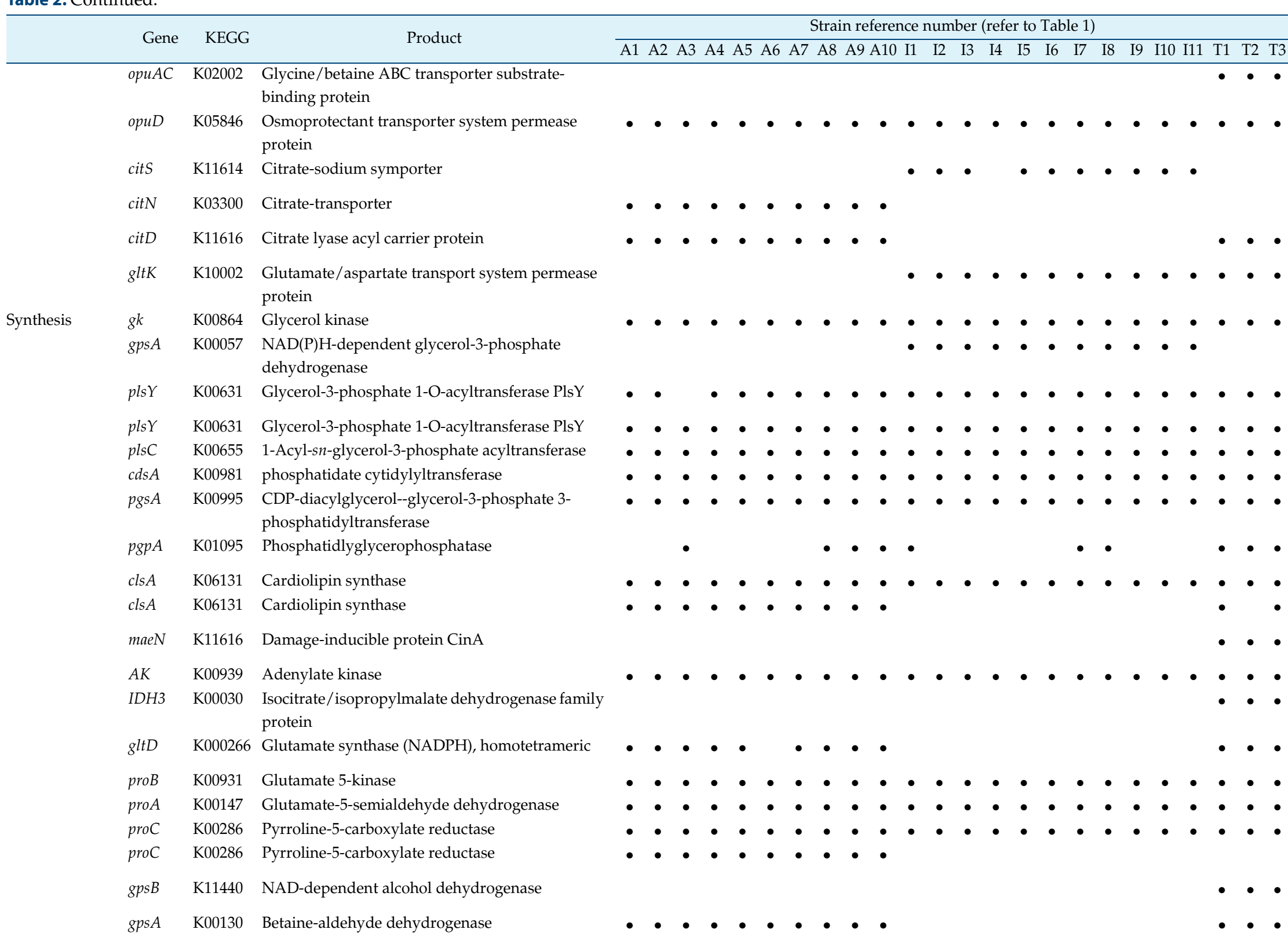


with E. faecium, and E. faecalis grew better than E. faecium in the presence of $7 \% \mathrm{NaCl}$. T. halophilus had a faster growth rate than the other two species in the presence of $7 \% \mathrm{NaCl}$, and was the only strain to grow in the presence of $14 \%$ $\mathrm{NaCl}$.

In conclusion, the genera Enterococcus and Tetragenococcus cannot readily be distinguished by phylogenetic analyses based on 16S rRNA gene sequences (Fig. S1) but show significant differences in salt tolerance. Here, comparative genomic analysis revealed several species-specific determinants of salt tolerance. The identification of such factors will help in the selection of appropriate starters for applications such as fermentation (i.e., low-salt or high-salt fermentation) used by the food industry.

\section{Acknowledgments}

This work was supported by the National Research Foundation of Korea (NRF) [NRF-2016R1D1A1B01011421 and NRF-2019R1A2C1003639]. We thank Dr. Jochen Blom from Justus-Liebig University for conducting EDGAR analysis, which was financially supported by BMBF grant FKZ031A533 within the de.NBI network. We also thank Tamsin Sheen, PhD, from Edanz Group (www.edanzediting. $\mathrm{com} / \mathrm{ac}$ ) for editing a draft of this manuscript.

\section{Conflict of Interest}

The authors have no financial conflicts of interest to declare.

\section{References}

1. Lee SS. 1995. Meju fermentation for a raw material of Korean traditional soy products. Kor. J. Mycol. 23: 161-175.

2. Yoo JY, Kim, HG. 1998. Characteristics of traditional mejus of nation-wide collection. J. Korean Soc. Food Sci. Nutr. 27: 259-267.

3. Shukla S, Lee JS, Park HK, Yoo JA, Hong SY, Kim JK, et al. 2015. Effect of novel starter culture on reduction of biogenic amines, quality improvement, and sensory properties of doenjang, a traditional Korean soybean fermented sauce variety. J. Food Sci. 80: M1794-1803.

4. Kum SJ, Yang SO, Lee SM, Chang PS, Choi YH, Lee JJ, et al. 2015. Effects of Aspergillus species inoculation and their enzymatic activities on the formation of volatile components in fermented soybean paste (doenjang). J. Agric. Food Chem. 63: 1401-1418.

5. Kim J-W, Doo H-S, Kwon T-H, Kim Y-S, Shin D-H. 2011. Quality characteristics of doenjang meju fermented with
Aspergillus species and Bacillus subtilis during fermentation. Korean J. Food Preserv. 18: 397-406.

6. Jeong DW, Heo S, Lee B, Lee H, Jeong K, Her JY, et al. 2017. Effects of the predominant bacteria from meju and doenjang on the production of volatile compounds during soybean fermentation. Int. J. Food Microbiol. 262: 8-13.

7. Jeong DW, Lee H, Jeong K, Kim CT, Shim ST, Lee JH. 2019. Effect of starter candidates and $\mathrm{NaCl}$ on the production of volatile compounds during soybean fermentation. J. Microbiol. Biotechnol. 29: 191-199.

8. Cho DH, Lee, WJ. 1970. Microbial studies of Korean native soy-sauce fermentation: a study on the microflora of fermented Korean maeju loaves. J. Korean Agric. Chem. Soc. 13: 35-42.

9. Kim KM, Lim J, Lee JJ, Hurh BS, Lee I. 2017. Characterization of Aspergillus sojae isolated from Meju, Korean traditional fermented soybean brick. J. Microbiol. Biotechnol. 27: 251-261.

10. Lee WJ, Cho, DH. 1971. Microbial studies of Korean native soy-sauce fermentation: a study on the microflora changes during Korean native soy-sauce fermentation. J. Korean Agric. Chem. Soc. 14: 137-148.

11. Kim YS, Jeong, DY, Hwang YT, Uhm T-B. 2011. Bacterial community profiling during the manufacturing process of traditional soybean paste by pyrosequencing method. Korean J. Microbiol. 47: 275-280.

12. Cho KM, Seo, WT. 2007. Bacterial diversity in Korean traditional soybean fermented foods (doenjang and ganjang) by $16 \mathrm{~S}$ rRNA gene sequence analysis. Food Sci. Biotechnol. 16: 320-324.

13. Kim TW, Lee JH, Kim SE, Park MH, Chang HC, Kim HY. 2009. Analysis of microbial communities in doenjang, a Korean fermented soybean paste, using nested PCR-denaturing gradient gel electrophoresis. Int. J. Food Microbiol. 131: 265271.

14. Kim YS, Kim MC, Kwon SW, Kim SJ, Park IC, Ka JO, et al. 2011. Analyses of bacterial communities in meju, a Korean traditional fermented soybean bricks, by cultivation-based and pyrosequencing methods. J. Microbiol. 49: 340-348.

15. Lee JH, Kim TW, Lee H, Chang HC, Kim HY. 2010. Determination of microbial diversity in meju, fermented cooked soya beans, using nested PCR-denaturing gradient gel electrophoresis. Lett. Appl. Microbiol. 51: 388-394.

16. Lee JH, Shin D, Lee B, Lee H, Lee I, Jeong DW. 2017. Genetic diversity and antibiotic resistance of Enterococcus faecalis isolates from traditional Korean fermented soybean foods. J. Microbiol. Biotechnol. 27: 916-924.

17. Euzeby JP. 2011. Efinitaions and abbreviations. in: List of Bacteria Names with Standing in Nomenclature. (ed.) J.P. Euzeby.

18. Gardiner GE, Ross RP, Wallace JM, Scanlan FP, Jagers PP, Fitzgerald GF, et al. 1999. Influence of a probiotic adjunct culture of Enterococcus faecium on the quality of cheddar cheese. J. Agric. Food Chem. 47: 4907-4916. 
19. Bhardwaj A, Malik RK, Chauhan P. 2008. Functional and safety aspects of enterococci in dairy foods. Indian $\mathrm{J}$. Microbiol. 48: 317-325.

20. Santos MM, Piccirillo C, Castro PM, Kalogerakis N, Pintado ME. 2012. Bioconversion of oleuropein to hydroxytyrosol by lactic acid bacteria. World J. Microbiol. Biotechnol. 28: 24352440.

21. Hanagata H, Shida O, Takagi H. 2003. Taxonomic homogeneity of a salt-tolerant lactic acid bacteria isolated from shoyu mash. J. Gen. Appl. Microbiol. 49: 95-100.

22. Bergey DH. 2009. Bergey's Manual of Systematic Bacteriology, pp. 594-623 Ed. Springer-Verlag New York.

23. Jeong DW, Kim HR, Jung G, Han S, Kim CT, Lee JH. 2014. Bacterial community migration in the ripening of doenjang, a traditional Korean fermented soybean food. J. Microbiol. Biotechnol. 24: 648-660.

24. Jeong MR, Jeong DW, Lee JH. 2015. Safety and biotechnological properties of Enterococcus faecalis and Enterococcus faecium isolates from Meju. J. Korean Soc. Appl. Biol. Chem. 58: 813-820.

25. Jeong DW, Heo S, Lee JH. 2017. Safety assessment of Tetragenococcus halophilus isolates from doenjang, a Korean high-salt-fermented soybean paste. Food Microbiol. 62: 92-98.

26. Lee JH, Heo S, Jeong K, Lee B, Jeong DW. 2018. Genomic insights into the non-histamine production and proteolytic and lipolytic activities of Tetragenococcus halophilus KUD23. FEMS Microbiol. Lett. 365: fnx252.

27. Facklam RR, Carvalho MG, Teixeira LM. 2002. Enterococcus, pp. 1-54. In Gilmore MS, Clewel DB, Courvalin P, Dunny GM, Murray BE, Rice LB (ed.), The Enterococci: Pathogenesis, Molecular Biology, and Antibiotic Resistance Ed. ASM Press, Washington, DC

28. Goris J, Konstantinidis KT, Klappenbach JA, Coenye T, Vandamme P, Tiedje JM. 2007. DNA-DNA hybridization values and their relationship to whole-genome sequence similarities. Int. J. Syst. Evol. Microbiol. 57: 81-91.

29. Aziz RK, Bartels D, Best AA, DeJongh M, Disz T, Edwards RA, et al. 2008. The RAST Server: rapid annotations using subsystems technology. BMC Genomics 9: 75.

30. Blom J, Albaum SP, Doppmeier D, Puhler A, Vorholter FJ, Zakrzewski M, et al. 2009. EDGAR: a software framework for the comparative analysis of prokaryotic genomes. BMC Bioinformatics 10: 154.

31. Wood JM, Bremer E, Csonka LN, Kraemer R, Poolman B, van der Heide $T$, et al. 2001. Osmosensing and osmoregulatory compatible solute accumulation by bacteria. Comp. Biochem. Physiol. A Mol. Integr. Physiol. 130: 437-460.

32. Epstein W. 2003. The roles and regulation of potassium in bacteria. Prog. Nucleic Acid Res. Mol. Biol. 75: 293-320.
33. Kraegeloh A, Amendt B, Kunte HJ. 2005. Potassium transport in a halophilic member of the bacteria domain: identification and characterization of the $\mathrm{K}+$ uptake systems TrkH and TrkI from Halomonas elongata DSM 2581T. J. Bacteriol. 187: 1036-1043.

34. Roberts MF. 2005. Organic compatible solutes of halotolerant and halophilic microorganisms. Saline Systems. 1: 5.

35. Whatmore AM, Chudek JA, Reed RH. 1990. The effects of osmotic upshock on the intracellular solute pools of Bacillus subtilis. J. Gen. Microbiol. 136: 2527-2535.

36. Holtmann G, Bakker EP, Uozumi N, Bremer E. 2003. KtrAB and KtrCD: two $\mathrm{K}+$ uptake systems in Bacillus subtilis and their role in adaptation to hypertonicity. J. Bacteriol. 185: 1289-1298.

37. Schwan WR, Wetzel KJ. 2016. Osmolyte transport in Staphylococcus aureus and the role in pathogenesis. World J. Clin. Infect. Dis. 6: 22-27.

38. Hoffmann T, Bremer E. 2017. Guardians in a stressful world: the Opu family of compatible solute transporters from Bacillus subtilis. Biol. Chem. 398: 193-214.

39. Chun BH, Han DM, Kim KH, Jeong SE, Park D, Jeon CO. 2019. Genomic and metabolic features of Tetragenococcus halophilus as revealed by pan-genome and transcriptome analyses. Food Microbiol. 83: 36-47.

40. Lin J, Zhu Y, Tang H, Yan J, Luo L. 2019. Identification of a GntR family regulator BusRTha and its regulatory mechanism in the glycine betaine $\mathrm{ABC}$ transport system of Tetragenococcus halophilus. Extremophiles 23: 451-460.

41. Boch J, Kempf B, Schmid R, Bremer E. 1996. Synthesis of the osmoprotectant glycine betaine in Bacillus subtilis: characterization of the gbs $A B$ genes. J. Bacteriol. 178: 51215129.

42. Romantsov T, Guan Z, Wood JM. 2009. Cardiolipin and the osmotic stress responses of bacteria. Biochim. Biophys. Acta 1788: 2092-2100.

43. Kanemasa Y, Yoshioka T, Hayashi H. 1972. Alteration of the phospholipid composition of Staphylococcus aureus cultured in medium containing $\mathrm{NaCl}$. Biochim. Biophys. Acta 280: 444450.

44. Tsai M, Ohniwa RL, Kato Y, Takeshita SL, Ohta T, Saito S, et al. 2011. Staphylococcus aureus requires cardiolipin for survival under conditions of high salinity. BMC Microbiol. 11: 13.

45. Jeong DW, Heo S, Ryu S, Blom J, Lee JH. 2017. Genomic insights into the virulence and salt tolerance of Staphylococcus equorum. Sci. Rep. 7: 5383.

46. He G, Wu C, Huang J, Zhou R. 2017. Effect of exogenous proline on metabolic response of Tetragenococcus halophilus under salt stress. J. Microbiol. Biotechnol. 27: 1682-1691. 